\title{
THEORY OF GROUP REPRESENTATIONS AND APPLICATIONS
}




\title{
THEORY OF GROUP REPRESENTATIONS AND APPLICATIONS
}

\author{
ASIM O. BARUT \\ Institute for Theoretical Physics, University of Colorado, \\ Boulder, Colo., U.S.A.
}

RYSZARD RĄCZKA

Institute for Nuclear Research, Warszawa, Polska

Second revised edition

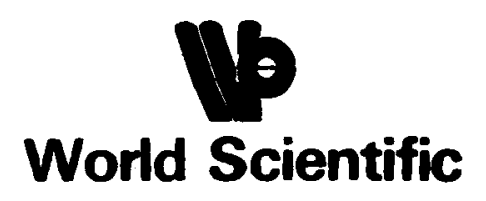




\section{Published by}

World Scientific Publishing Co. Pte. Ltd.

P O Box 128, Farrer Road, Singapore 912805

USA office: Suite 1B, 1060 Main Street, River Edge, NJ 07661

UK office: 57 Shelton Street, Covent Garden, London WC2H 9HE

Graphic design: Zygmunt Ziemka

Motif from Sky and water I, the graphic work of M. C. Escher

Library of Congress Cataloging-in-Publication data is available.

First published 1986

Reprinted 2000

\section{THEORY OF GROUP REPRESENTATIONS AND APPLICATIONS}

Copyright $\odot 1986$ by World Scientific Publishing Co. Pte. Ltd.

All rights reserved. This book, or parts thereof, may not be reproduced in any form or by any means. electronic or mechanical, including photocopying, recording or any information storage and retrieval system now known or to be invented, without written permission from the Publisher.

For photocopying of material in this volume, please pay a copying fee through the Copyright Clearance Center, Inc., 222 Rosewood Drive, Danvers, MA 01923, USA. In this case permission to photocopy is not required from the publisher.

ISBN 9971-50-216-X

ISBN 9971-50-217-8 (pbk)

Printed in Singapore by Uto-Print 


\section{Dedicated to}

\section{OLA and PIERRETTE}




\section{Preface}

This book is written primarily for physicists, but also for other scientists and mathematicians to acquaint them with the more modern and powerful methods and results of the theory of topological groups and of group representations and to show the remarkable wide scope of applications. In this respect it is markedly different than, and goes much beyond, the standard books on group theory in quantum mechanics. Although we aimed at a mathematically rigorous level, we tried to make the exposition very explicit, the language less abstract, and have illustrated the results by many examples and applications.

During the past two decades many investigations by physicists and mathematicians have brought a certain degree of maturity and completeness to the theory of group representations. We have in mind the new results in the development of the general theory, as well as many explicit constructions of representations of specific groups. At the same time new applications of, in particular, noncompact groups revealed interesting structures in the symmetry, as well as in the dynamics, of quantum theory. The mathematical sophistication and knowledge of the physicists have also markedly increased. For all these reasons it is timely to collect the new results and to present a book on a much higher level than before, in order to facilitate further developments and applications of group representations.

There is no other comparable book on group representations, neither in mathematical nor in physical literature, and we hope that it will prove to be useful in many areas of research.

Many of the results appear, to our knowledge, for the first time in book form. These include, in particular, a systematic exposition of the theory and applications of induced representations, the classification of all finite-dimensional irreducible representations of arbitrary Lie groups, the representation theory of Lie and enveloping algebras by means of unbounded operators, new integrability conditions for representations of Lie algebras and harmonic analysis on homogeneous spaces.

In the domain of applications, we have discussed the general problem of symmetries in quantum theory, in particular, relativistic invariance, group theoretical derivation of relativistic wave equations, as well as various applications of group representations to dynamical problems in quantum theory. 
We have tried to achieve a certain amount of completeness so that the book can be used as a textbook for an advanced course in mathematical physics on Lie algebras, Lie groups and their representations. Some of the standard topics can be found scattered in various texts but, so far, not all under a single cover.

A book in the border area of theoretical physics and pure mathematics is always problematic. And so this book may seem to be too difficult, detailed and abstract to some physicists, and not detailed and complete enough for some mathematicians, as we have deliberately omitted a number of proofs. Fortunately the demand for knowledge of modern mathematics among physicists is on the rise. And to give the proofs of all theorems in such a wide area of mathematics is impossible even in a large volume as this one. Where too long technical details would cloud the clarity and when the steps of the proof did not seem to be essential for further development of the subject we have omitted the proofs.

The material collected in this book originated from lectures given by the authors over many years in Warsalv, Trieste, Schladming, Istanbul, Göteborg and Boulder. It has passed several rewritings. We are especially grateful to many friends and colleagues who read, corrected and commented on parts of the manuscript. We would like to thank Dr. S. Woronowicz for his careful and patient reading of the entire manuscript and pointing out numerous improvements and corrections. We have discussed parts of the manuscript with many of our friends and colleagues who made constructive criticism, in particular S. Dymus, M. Flato, B. Kostant, G. Mackey, K. Maurin, L. Michel, I. Segal, D. Sternheimer, S. Ström, A. Sym, I. Szczyrba and A. Wawrzyńczyk.

A considerable part of this book contains the results of the research carried out under collaboration between Colorado University in Boulder and Institute for Nuclear Research in Warsaw. This collaboration was partially supported by National Science Foundation under the contract No. GF-41958. The authors are particularly grateful to Dr. C. Zalar, Program Manager for Europe and North America for his kind and effective support for American-Polish scientific collaboration.

Finally, we would like to express our gratitude to Mr J. Panz, editor in the Polish Scientific Publishers, for his great help in preparing this manuscript for printing. We are also obliged to Mrs Z. Osek for her kind help in all phases of preparing the manuscript for publication.

A. O. Barut and R. Raczka

Boulder and Warsaw, August 1976 
Contents

PREface .. . . . . . . . . . . . . . . . . . . . . . . . VII

OUTLINE OF THE BOOK. . . . . . . . . . . . . . . . . . . . . . XV

Notations . . . . . . . . . . . . . . . . . . . . . . . . . . XIX

Chapter 1

LiE Algebras

$\S 1$. Basic Concepts and General Properties . . . . . . . . . . . 1

§ 2. Solvable, Nilpotent, Semisimple and Simple Lie Algebras . . . . 10

$\S 3$. The Structure of Lie Algebras . . . . . . . . . . . . . . . . . 17

$\S 4$. Classification of Simple, Complex Lie Algebras . . . . . . . . . 20

§5. Classification of Simple, Real Lie Algebras . . . . . . . . . . . 29

\$6. The Gauss, Cartan and Iwasawa Decompositions . . . . . . . 37

$\S 7$. An Application. On Unification of the Poincaré Algebra and Internal Symmetry Algebra . . . . . . . . . . . . . . . 43

$\S 8$. Contraction of Lie Algebras . . . . . . . . . . . . . . . . . . 44

\$. Comments and Supplements . . . . . . . . . . . . . . . . . . 46

$\S 10$. Exercises . . . . . . . . . . . . . . . . . . . . . . 48

Chapter 2

TOPOLOGICAL Groups

$\S 1$. Topological Spaces . . . . . . . . . . . . . . . . . . . . 52

$\S 2$. Topological Groups . . . . . . . . . . . . . . . . . . . . 61

$\S 3$. The Haar Measure . . . . . . . . . . . . . . . . . . . . . . 67

$\S 4$. Comments and Supplements . . . . . . . . . . . . . . . . . 70

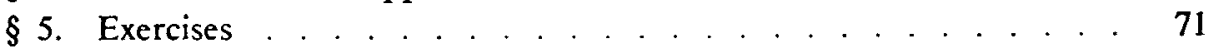

CHAPTER 3

LIE GROUPS

$\S 1$. Differentiable Manifolds . . . . . . . . . . . . . . . . 75

$\S 2$. Lie Groups . . . . . . . . . . . . . . . . . . . . . . . . . 81

$\S 3$. The Lie Algebra of a Lie Group . . . . . . . . . . . . . . . . 85

$\S$ 4. The Direct and Semidirect Products . . . . . . . . . . . . . 95

§ 5. Levi-Malcev Decomposition . . . . . . . . . . . . . . . . . . 98

§. Gauss, Cartan, Iwasawa and Bruhat Global Decompositions . . . . 100

$\S 7$. Classification of Simple Lie Groups . . . . . . . . . . . . . . . 106

$\S 8$. Structure of Compact Lie Groups . . . . . . . . . . . . . 108 
§ 9. Invariant Metric and Invariant Measure on Lie Groups . . . . . 109

$\$ 10$. Comments and Supplements . . . . . . . . . . . . . . . . . . 111

$\S 11$. Exercises . . . . . . . . . . . . . . . . . . . . . . . . . . 114

CHAPTER 4

Homogeneous aNd Symmetric SPACES

$\S 1$. Homogeneous Spaces . . . . . . . . . . . . . . . . . . . . 123

§ 2. Symmetric Spaces . . . . . . . . . . . . . . . . . . . . . . 124

\$ 3. Invariant and Quasi-Invariant Measures on Homogeneous Spaces 128

\$ 4. Comments and Supplements . . . . . . . . . . . . . . . . . . 132

§. Exercises . . . . . . . . . . . . . . . . . . . . . 132

ChAPTER 5

Group RePresentations

$\S 1$. Basic Concepts . . . . . . . . . . . . . . . . . . . . . . . . 134

§ 2. Equivalence of Representations . . . . . . . . . . . . . . . . 139

$\S 3$. Irreducibility and Reducibility. . . . . . . . . . . . . . . . . 141

§4. Cyclic Representations . . . . . . . . . . . . . . . . . . . . 145

§ 5. Tensor Product of Representations . . . . . . . . . . . . . . . 147

§ 6. Direct Integral Decomposition of Unitary Representations . . . . 150

§ 7. Comments and Supplements . . . . . . . . . . . . . . . . . . 156

§. Exercises . . . . . . . . . . . . . . . . . .

CHAPTER 6

Representations of Commutative Groups

$\S 1$. Irreducible Representations and Characters . . . . . . . . . . . 159

$\S 2$. Stone and SNAG Theorems . . . . . . . . . . . . . . . . 160

§3. Comments and Supplements . . . . . . . . . . . . . . . . . 163

§4. Exercises . . . . . . . . . . . . . . . . . . . . . . . . . . 164

CHAPTER 7

Representations of Compact Groups

$\S 1$. Basic Properties of Representations of Compact Groups . . . . . 166

$\S 2$ 2. Peter-Weyl and Weyl Approximation Theorems . . . . . . . . . 172

§ 3. Projection Operators and Irreducible Representations . . . . . . . . 177

§ 4. Applications . . . . . . . . . . . . . . . . . . . . . . . . 179

§ 5. Representations of Finite Groups . . . . . . . . . . . . . . . 186

§6. Comments and Supplements . . . . . . . . . . . . . . . . . . 195

§ 7. Exercises . . . . . . . . . . . . . . . . . . . . . . . . . . 197

CHAPTER 8

Finite-Dimensional Representations of Lie Groups

$\S 1$. General Properties of Representations of Solvable and Semisimple Lie Groups 
§ 2. Induced Representations of Lie Groups . . . . . . . . . . . 205

$\S 3$. The Representations of $\mathrm{GL}(n, C), \mathrm{GL}(n, R), U(p, q), U(n), \mathrm{SL}(n, C)$, $\mathrm{SL}(n, R), \mathrm{SU}(p, q)$, and $\mathrm{SU}(n) \ldots \ldots . . . . . . .213$

§4. The Representations of the Symplectic Groups $\operatorname{Sp}(n, C), \operatorname{Sp}(n, R)$ and $\operatorname{Sp}(n) \ldots \ldots \ldots \ldots$. . . . . . . . . 217

$\S 5$. The Representations of Orthogonal Groups $\mathrm{SO}(n, C), \mathrm{SO}(p, q), \mathrm{SO}^{*}(n)$, and $S O(n)$.................. 219

$\S 6$. The Fundamental Representations . . . . . . . . . . . . . 223

§7. Representations of Arbitrary Lie Groups . . . . . . . . . . . . 225

$\S 8$. Further Results and Comments . . . . . . . . . . . . . . . 227

§ 9. Exercises . . . . . . . . . . . . . . . . . . 238

ChAPTER 9

Tensor Operators, Enveloping Algebras and Enveloping Fields

$\S 1$. The Tensor Operators . . . . . . . . . . . . . . . . 242

$\S 2$. The Enveloping Algebra . . . . . . . . . . . . . . 249

$\S 3$. The Invariant Operators . . . . . . . . . . . . . . . . 251

$\$$ 4. Casimir Operators for Classical Lie Group . . . . . . . . . . . 254

§5. The Enveloping Field . . . . . . . . . . . . . . . . . 266

§6. Further Results and Comments . . . . . . . . . . . . . . . . 273

$\S 7$. Exercises ................. 275

Chapter 10

The Explicit Construction of Finite-Dimensional Irreducirle REPRESENTATIONS

$\S 1$. The Gel'fand-Zetlin Method . . . . . . . . . . . . . . . . 277

§ 2. The Tensor Method ... . . . . . . . . . . . . . . . 291

$\S 3$. The Method of Harmonic Functions . . . . . . . . . . . . . 302

$\S$ 4. The Method of Creation and Annihilation Operators . . . . . . 309

$\S 5$. Comments and Supplements ..... . . . . . . . . 312

§6. Exercises ................... 314

Chapter 11

RePresentation Theory of Lie AND ENVELOPING Algebras by UNBOUNDED OPERATORS: ANALYTIC VECTORS AND INTEGRABILITY

§1. Representations of Lie Algebras by Unbounded Operators . . . . 318

\$ 2. Representations of Enveloping Algebras by Unbounded Operators 323

§ 3. Analytic Vectors and Analytic Dominance . . . . . . . . . . . . 331

§ 4. Analytic Vectors for Unitary Representations of Lie Groups . . . . 344

$\S 5$. Integrability of Representations of Lie Algebras . . . . . . . . . 348

\$ 6. $F S^{3}$-Theory of Integrability of Lie Algebras Representations . . . . 352

$\S 7$. The 'Heat Equation' on a Lie Group and Analytic Vectors . . . . 358 
§ 8. Algebraic Construction of Irreducible Representations . . . . . . 365

§ 9. Comments and Supplements . . . . . . . . . . . . . . 372

$\S 10$. Exercises . . . . . . . . . . . . . . . . . . . . 373

Chapter 12

Quantum Dynamical Applications of lie Algebra Representations

§ 1. Symmetry Algebras in Hamiltonian Formulation . . . . . . . . . 378

$\S 2$. Dynamical Lie Algebras . . . . . . . . . . . . . . . . . . 382

$\S 3$. Exercises . . . . . . . . . . . . . . . . . 386

CHAPTER 13

Group Theory and Group Representations in Quantum Theory

$\S 1$. Group Representations in Physics . . . . . . . . . . . . . . . 392

$\S 2$. Kinematical Postulates of Quantum Theory . . . . . . . . . . . 394

$\S 3$. Symmetries of Physical Systems . . . . . . . . . . . . . . . . 406

$\S 4$. Dynamical Symmetries of Relativistic and Non-Relativistic Systems 412

§ 5. Comments and Supplements . . . . . . . . . . . . . . . . . . 417

$\S 6$. Exercises . . . . . . . . . . . . . . . . . . . 418

Chapter 14

Harmonic Analysis on lie Groups. Special functions and Group REPRESENTATIONS

$\S 1$ Harmonic Analysis on Abelian and Compact Lie Groups . . . . . 421

$\S 2$. Harmonic Analysis on Unimodular Lie Groups . . . . . . . . . 423

$\S 3$. Harmonic Analysis on Semidirect Product of Groups . . . . . . . . 431

$\S 4$. Comments and Supplements . . . . . . . . . . . . . . . . . 435

$\S 5$. Exercises

Chapter 15

Harmonic ANalysis on Homogeneous Spaces

$\S 1$. Invariant Operators on Homogeneous Spaces . . . . . . . . . . 439

§ 2. Harmonic Analysis on Homogeneous Spaces . . . . . . . . . . 441

§3. Harmonic Analysis on Symmetric Spaces Associated with PseudoOrthogonal Groups $\mathrm{SO}(p, q)$. . . . . . . . . . . . . . . 446

§ 4. Generalized Projection Operators . . . . . . . . . . . . . . . 459

§ 5. Comments and Supplements . . . . . . . . . . . . . . . . . . 466

§6. Exercises . . . . . . . . . . . . . . . . . . . . . 470

ChAPTER 16

INDUCED REPRESENTATIONS

§ 1. The Concept of Induced Representations . . . . . . . . . . . . 473

§ 2. Basic Properties of Induced Representation . . . . . . . . . . . 487 
§ 3. Systems of Imprimitivity . . . . . . . . . . . . . . . . . . . 493

\$4. Comments and Supplements . . . . . . . . . . . . . . . . . . 501

$\S 5$. Exercises . . . . . . . . . . . . . . . . . . . . 493

CHAPTER 17

INDUCED RePresentations of SEMIDIReCt Products

$\S 1$. Representation Theory of Semidirect Products . . . . . . . . . . . 503

$\S 2$. Induced Unitary Representations of the Poincaré Group . . . . . 513

§ 3. Representation of the Extended Poincaré Group . . . . . . . . . 525

\$ 4. Indecomposable Representations of Poincaré Group . . . . . . . 527

$\S 5$. Comments and Supplements . . . . . . . . . . . . . . . . . . 536

§6. Exercises . . . . . . . . . . . . . . . . . . 537

Chapter 18

Fundamental ThEOREMS OF INDUCED REPRESENTATIONS

$\S 1$. The Induction-Reduction Theorem . . . . . . . . . . . . . . . 540

$\S 2$. Tensor-Product Theorem . . . . . . . . . . . . . . . . . . . 546

$\S 3$. The Frobenius Reciprocity Theorem . . . . . . . . . . . . . . 549

§ 4. Comments and Supplements . . . . . . . . . . . . . . . . . . 553

$\S 5$. Exercises . . . . . . . . . . . . . . . . . . . . 553

ChAPTER 19

Induced Representations of Semisimple Lie Groups

$\S 1$. Induced Representations of Semisimple Lie Groups . . . . . . . . 555

$\S 2$. Properties of the Group $\operatorname{SL}(n, C)$ and Its Subgroups . . . . . . . 559

$\S 3$. The Principal Nondegenerate Series of Unitary Representations of $\mathrm{SL}(n, C)$. . . . . . . . . . . . . . . . . . . 560

$\S 4$. Principal Degenerate Series of $\operatorname{SL}(n, C)$. . . . . . . . . . . 567

$\S 5$. Supplementary Nondegenerate and Degenerate Series . . . . . . 570

$\S 6$. Comments and Supplements . . . . . . . . . . . . . . . . . . 577

$\$ 7$. Exercises . . . . . . . . . . . . . . . . . . . . . . 578

ChAPTER 20

APPLICATIONS OF INDUCED REPRESENTATIONS

$\S 1$. The Relativistic Position Operator . . . . . . . . . . . . . . 581

$\S 2$. The Representations of the Heisenberg Commutation Relations . . 588

$\S 3$. Comments and Supplements . . . . . . . . . . . . . . . . 591

$\S 4$. Exercises . . . . . . . . . . . . . . . . . . . . 593

ChAPTER 21

Group Representations in Relativistic Quantum Theory

$\S 1$. Relativistic Wave Equations and Induced Representations . . . . 596

$\S 2$. Finite Component Relativistic Wave Equations . . . . . . . . . 601 
§ 3. Infinite Component Wave Equations . . . . . . . . . . . . . . 609

\$4. Group Extensions and Applications . . . . . . . . . . . . . . 619

§ 5. Space-Time and Internal Symmetries . . . . . . . . . . . . . . 626

\$6. Comments and Supplements . . . . . . . . . . . . . . . . . . 630

§ 7. Exercises . . . . . . . . . . . . . . . . 636

APPENDIX A

Algebra, TOPOlOGY, MEASURE AND INTEGRATION THEORY . . . . . . . 637

APPENDIX B

Functional ANalysis

$\S 1$. Closed, Symmetric and Self-Adjoint Operators in Hilbert Space . . 641

$\S 2$. Integration of Vector and Operator Functions . . . . . . . . . . 645

§ 3. Spectral Theory of Operators . . . . . . . . . . . . . . . . . 649

$\S 4$. Functions of Self-Adjoint Operators . . . . . . . . . . . . . . 662

$\S 5$. Essentially Self-Adjoint Operators . . . . . . . . . . . . . 663

BIBLIOGRAPHY . . . . . . . . . . . . . . . . . 667

LIST OF IMPORTANT SYMBOLS . . . . . . . . . . . . . . . . . . . . 703

AUTHOR INDEX . . . . . . . . . . . . . . . . . . . . . 706

SUBUECT INDEX 


\section{Outline of the Book}

The book begins with a long chapter on Lie algebras. This is a self-contained detailed exposition of the theory and applications of Lie algebras. The theory of Lie algebras is an independent discipline in its own right and the chapter can be read independently of others. We give, after basic concepts, the structure and theory of arbitrary Lie algebras, a description of nilpotent and solvable algebras and a complete classification of both complex and real simple Lie algebras. Another feature is the detailed discussion of decomposition theorems of Lie algebras, i.e., Gauss, Cartan and Iwasawa decompositions.

Ch. 2 begins with a review of the properties of topological spaces, in order to introduce the concepts of topological groups. The general properties of topological groups such as compactness, connectedness and metric properties are treated. We discuss further integration over the group manifold, i.e., the invariant measure (Haar measure) on the group. The fundamental Mackey decomposition theorem of topological groups is also given.

Ch. 3 begins with a review of differentiable manifolds, their analytic structures and tangent spaces. With these preparations on topological groups and differentiable manifolds we introduce Lie groups as topological groups with an analytic structure and derive the basic relations between Lie groups and Lie algebras. The remaining sections of ch. 3 are devoted to the composition and decomposition properties of groups (i.e., Levi-Malcev, Gauss, Cartan, Iwasawa decompositions), to the classification of Lie groups and to some results on the structure of Lie groups and to the construction of invariant measure and of invariant metric.

In the next chapter, 4, we introduce the concepts of homogeneous and symmetric spaces on which groups act. These concepts play an important role in the modern theory of group representations and in physical applications. We further give a classification of globally symmetric Riemannian spaces associated with the classical simple Lie groups. Also discussed in this chapter is the concept of quasi-invariant measure, because invariant measures do not exist in general on homogeneous spaces.

The theory of group representations, the main theme of the book, begins in ch. 5 where we first give the definitions, the general properties of representations, irreducibility, equivalence, tensor and direct product of representations. We further treat the Mautner and the Gel'fand-Raikov theorems on the decomposition and completeness of group representations. 
The detailed group representation theory is then developed in successive steps beginning with the simplest case of commutative groups, in ch. 6 , followed by the representations of compact groups, in ch. 7. For completeness we also review here, as a special case, the representations of finite groups. The representation theory of compact groups is complete and we give the general theorems (the Peter-Weyl and Weyl approximation theorems) of this theory. With a view towards applications, we discuss also the projection operators, decomposition of the representations and of tensor products.

Next comes the description of all finite-dimensional irreducible representations of arbitrary Lie groups (compact or non-compact) (ch. 8). Here we give a more complete treatment of the properties of representations of semisimple groups than is available, to our knowledge, in any other book. The methods for the explicit construction of the finite-dimensional representations are treated in ch. 10, after a necessary discussion of tensor operators, enveloping algebras and invariant or Casimir operators and their spectra in ch. 9. (These concepts are used to specify and label the representations.) Among the methods we give the Gel'fand-Zetlin method, the tensor method, the method of harmonic functions and the method of creation and annihilation operators.

Ch. 11 deals with the representation theory of Lie and enveloping algebras by unbounded operators and the related questions of integrability of Lie algebra representations to the representations of the corresponding Lie groups. This is one of the most important chapters of the book. The theory of unbounded operators is also important for applications because most of the observables in quantum theory are represented by unbounded operators. More specifically, the theory of analytic vectors for Lie groups and Lie algebras is presented.

In chs. 12 and 13 we give a treatment of the role that the theory of group representation plays in all areas of quantum theory and specific applications. The mathematical structure of group representations in the Hilbert space is particularly adapted to quantum theory: In fact, we can base the framework of quantum theory solely on the concept of group representations. Historically, also, the concepts of Hilbert space and representation of groups in the Hilbert space had their origin in quantum theory. We also discuss the concepts of kinematical and dynamical symmetries, a classification of basic symmetries of physics and the use of group representations in solving dynamical problems in quantum mechanics.

The next two chapters (14 and 15) are devoted to harmonic analysis on Lie groups and on homogeneous and symmetric spaces. Here the theory encompasses a generalization of the Fourier expansion for non-commutative groups, the corresponding spectral synthesis and Plancherel formulas. We discuss the general theory as well as specific applications to some simple and semi-direct product groups.

The following four chapters, 16-19, are devoted to the theory of induced 
representations, one of the most important themes of the book. Already in ch. 8 we have used induced representations to obtain a classification as well as the explicit form of all irreducible finite-dimensional representations of Lie groups. Here the general theory is presented.

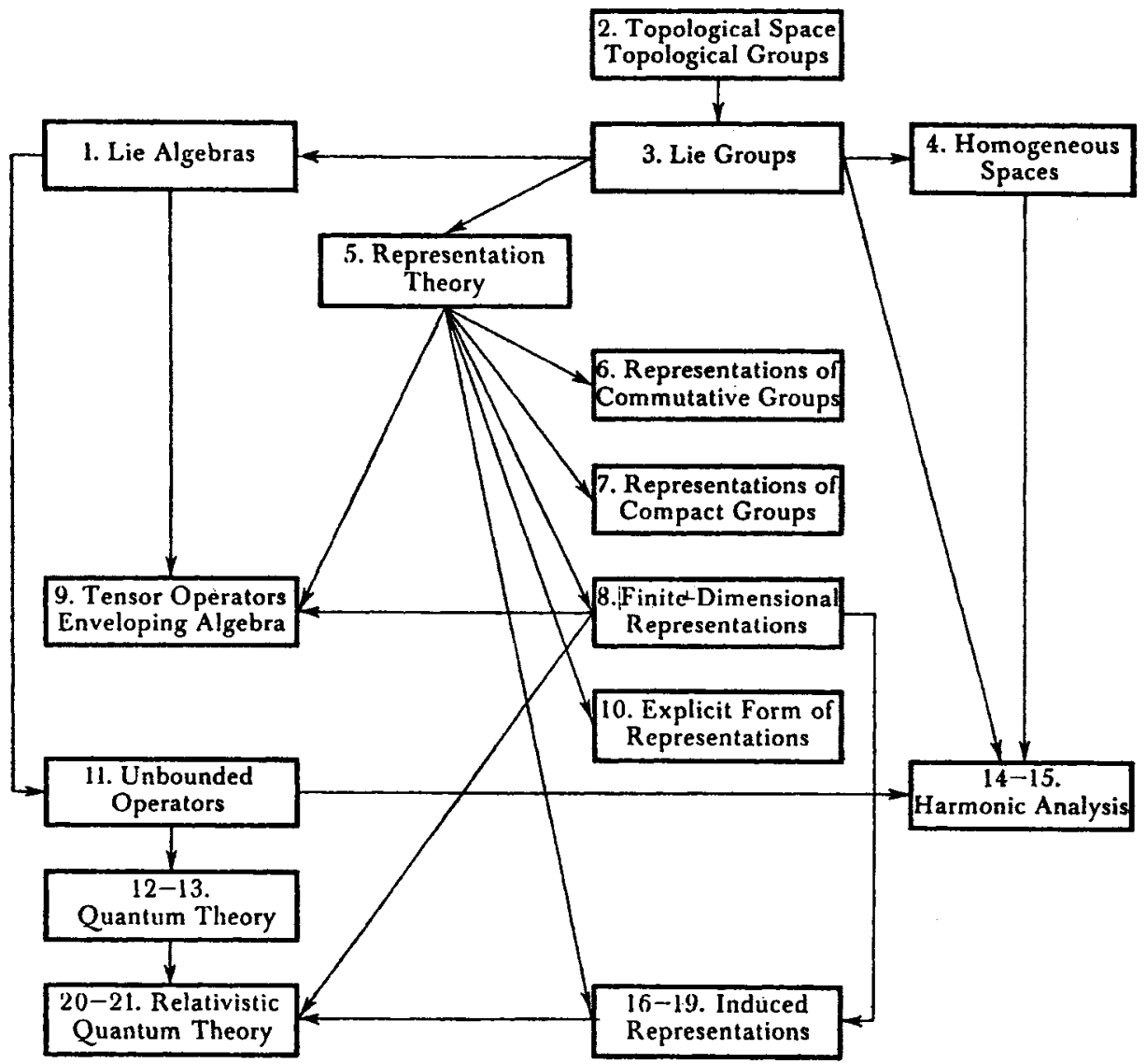

Ch. 16 deals with the basic properties of induced representations and the fundamental imprimitivity theorem. In the next chapter, 17, the induced representations of semi-direct product of groups is given, with a derivation of the complete classification of all representations of the Poincare group. The further properties of induced representations (the induction-reduction theorem, the tensor product theorem and the Frobenius reciprocity theorem) are discussed in ch. 18. In ch. 19 the theory is applied to derive explicitly the induced irreducible unitary, hence infinite-dimensional, representations of principal and supplementary series of complex classical Lie groups. 
Finally, in chs. 20-21, we take up applications of the imprimitivity theorem and induced representations of the Poincaré group in quantum physics: first to the concept of relativistic position operator and to the proof of equivalence of Heisenberg and Schrödinger descriptions in non-relativistic quantum mechanics (in ch. 20), next, in ch. 21, to the classification of all finite-dimensional relativistic wave equations, to applications of imaginary mass representations, to Gel'fand-Yaglom type and infinite component relativistic wave equations, and to the problem of group extension of the representations of the relativity group by discrete operations and by other symmetry groups.

A number of mathematical concepts which are not so familiar to physicists and which are essential for the book have been collected in the appendices on functional analysis, and on other results from algebra, topology, integration theory, etc.

Each chapter contains at the end notes on further developments of the subject as well as exercises. 


\section{Notations}

Our space-time metric $g_{\mu v}$ is such that $g_{00}=-g_{11}=-g_{22}=-g_{33}=1$. The symbol $a^{*}$ denotes the hermitian conjugation of a matrix or an operator $a$. The symbols $\bar{a}$ and $a^{T}$ denote the complex conjugation and the transposition of a matrix $a$. The symbol $\nabla$ denotes the end of the proof of a theorem or of an example. The direct sum of vector spaces $V_{i}$ is written as $V_{1}+V_{2} \dot{+} \ldots$ and the direct sum of Lie algebras $L_{i}$ as $L_{1} \oplus L_{2} \oplus \ldots$ The semidirect sum of two Lie algebras is denoted by $L_{1} \rightarrow L_{2}$ and the semidirect product of two groups as $G_{1} \gg G_{2}$, while the direct product of two groups is written as $G_{1} \times G_{2}$. The expression 'th. 8.6.3' means theorem 3 of chapter 8 in section 6. The expression 'exercise 9.7.3.1' means exercise $\S 3.1$ in chapter 9, section 7. The quotation, say 'Lunn $1969^{\prime}$, denotes the reference to the paper of the author Lunn from the year 1969: if there are several papers of the same author in a given year we have additional index $a, b, \ldots$, etc.

The symbol

$$
\left[\frac{1}{2} n\right]= \begin{cases}\frac{1}{2} n & \text { if } n=2 r \\ \frac{1}{2}(n-1) & \text { if } n=2 r+1\end{cases}
$$

the symbol

$$
\left\{\frac{1}{2} n\right\}= \begin{cases}\frac{1}{2} n & \text { if } n=2 r \\ \frac{1}{2}(n+1) & \text { if } n=2 r+1\end{cases}
$$

We use throughout Einstein summation convention unless stated otherwise. For the sake of simplicity we use the symbol $\sqrt{ }(\ldots)$ instead of $\sqrt{(\ldots)}$ for roots. 